\title{
Perspectives from Italy during the COVID-19 pandemic: nationwide survey-based focus on minimally invasive HPB surgery
}

\author{
Luca Aldrighetti ${ }^{1}$ (D - Ugo Boggi ${ }^{2} \cdot$ Massimo Falconi $^{3} \cdot$ Felice Giuliante $^{4} \cdot$ Federica Cipriani ${ }^{1} \cdot$ Francesca Ratti $^{1}$. \\ Guido Torzilli ${ }^{5}$ on behalf of Italian Association of HepatoBilioPancreatic Surgeons-AICEP
}

Received: 15 May 2020 / Accepted: 20 May 2020 / Published online: 29 May 2020

(C) Italian Society of Surgery (SIC) 2020

\begin{abstract}
The safety of minimally invasive procedures during COVID pandemic remains hotly debated, especially in a country, like Italy, where minimally invasive techniques have progressively and pervasively entered clinical practice, in both the hepatobiliary and pancreatic community. A nationwide snapshot of the management of HPB minimally invasive surgery activity during COVID-19 pandemic is provided: a survey was developed and conducted within AICEP (Italian Association of HepatoBilioPancreatic Surgeons) with the final aim of conveying the experience, knowledge, and opinions into a unitary report enabling more efficient crisis management. Results from the survey (81 respondents) show that, in Italian hospitals, minimally invasive surgery maintains its role despite the COVID-19 pandemic, with the registered reduction of cases being proportional to the overall reduction of the HPB surgical activity. Respondents agree that the switch from minimally invasive to open technique can be considered as a valid option for cases with a high technical complexity. Several issues merit specific attention: screening for virus positivity should be universally performed; only expert surgical teams should operate on positive patients and specific technical measures to lower the biological risk of contamination during surgery must be followed. Future studies specifically designed to establish the true risks in minimally invasive surgery are suggested. Furthermore, a standard and univocal process of prioritization of patients from Regional Healthcare Systems is advisable.
\end{abstract}

Keywords Liver $\cdot$ Pancreas $\cdot$ Minimally invasive $\cdot$ Pandemic $\cdot$ COVID-19

Members of "The Italian Association of HepatoBilioPancreatic Surgeons-AICEP” are listed in Acknowledgement section.

Electronic supplementary material The online version of this article (https://doi.org/10.1007/s13304-020-00815-5) contains supplementary material, which is available to authorized users.

Luca Aldrighetti

aldrighetti.luca@hsr.it

1 Hepatobiliary Surgery Division, IRCCS San Raffaele Hospital, Via Olgettina 60, 20132 Milan, Italy

2 Department of Transplant and General Surgery, University of Pisa, Pisa, Italy

3 Division of Pancreatic Surgery, IRCCS San Raffaele Hospital, Milan, Italy

4 Hepatobiliary Surgery Unit, Foundation "Policlinico Universitario A.Gemelli”, Catholic University, Rome, Italy

5 Division of Hepatobiliary and General Surgery, Department of Surgery, Humanitas University and Research Hospital, IRCCS, Rozzano, Milan, Italy

\section{Introduction}

The official number of COVID-19-related deaths in Italy is 28.884 (20 February-3 May 2020): this impressive figure represents the intense and unprecedented pressure that the National Healthcare System has been put under. Within the short period of 73 days, increased hospitalization demands for patients requiring respiratory support, rapid saturation of intensive care unit (ICU) beds, and the shift of medical (anesthesiologists above all) and nursing staff from elective to emergency clinical activity generated the need for resource optimization [1,2]. Subsequently, surgeons face challenges related to allocation and possible delays of curative surgery for oncological patients, especially for those bearing tumors with a narrow window for delivery of treatment [3-5], such as HPB tumors (i.e., pancreatic adenocarcinoma, perihilar cholangiocarcinoma, and colorectal metastases rescued after chemotherapy). On the other hand, the possibility of an increased risk of infection for surgical teams has been raised, in particular with the use of 
minimally invasive surgery (MIS), owing to possible exposure to SARS-CoV-2 virus spread by pneumoperitoneal carbon dioxide and smoke produced by energy devices [6]. Hence, the safety of minimally invasive procedures remains hotly debated, especially in a country, like Italy, where minimally invasive techniques have progressively and pervasively entered clinical practice, in both the hepatobiliary and pancreatic community.

Such a scenario urgently necessitates the gathering of a nationwide snapshot of the management of HPB minimally invasive surgery activity during COVID-19 pandemic. Therefore, a survey was developed and conducted within AICEP (Italian Association of HepatoBilioPancreatic Surgeons) with the final aim of conveying the experience, knowledge, and opinions into a unitary report enabling more efficient crisis management, by rapidly adopting strategies that are deemed useful, and also by filling any relevant gaps that existed previously.

\section{Materials and methods}

The survey was a self-administered electronic questionnaire developed by the survey administration application Google Forms (Google LCC, Mountain View, California, USA). It was designed by the promoting group (including the president of the AICEP, two pancreatic and two hepatic surgeons) and submitted by personal email addresses to members of the AICEP (Italian Association of HepatoBilioPancreatic Surgeons). Only one answer per team was allowed and all returned questionnaires were screened to eliminate any double replay, even if no apparent duplicate data were found. No minimal cutoff in surgical volume (both for liver and pancreas surgery) was established for inclusion in the survey. A surgeon at each center was identified for correspondence regarding incomplete or missing answers. No incentives were used to increase the response rate.

The online survey included 59 questions, organized in 6 sessions (general details; waiting list management and indications; preoperative assessment; intraoperative management; postoperative ward assistance of inpatients; human resources). Questions were close ended or multiple choice (with several questions allowing the possibility to choose multiple answers), while open questions were not present. A summary of the items of the survey, as well as answers obtained by centers, is provided in Tables 1 and 2 (the detailed questionnaire is available in the Additional Online Material).

The survey was conducted in the first week of April 2020, being closed on April 10th, 2020 after two reminds to non-respondents.
Table 1 HPB activity during the COVID-19 pandemic

\begin{tabular}{ll}
\hline Hub centers for COVID-19 & $67 / 81(82.7 \%)$ \\
Activity & \\
Hepatobiliary & $68 / 81(84 \%)$ \\
Pancreatic & $55 / 81(67.9 \%)$ \\
Reduction of activity & \\
No reduction & $8 / 81(9.9 \%)$ \\
$<50 \%$ & $14 / 81(17.3 \%)$ \\
$\geq 50 \%$ & $59 / 81(25.9 \%)$ \\
Reduction of MIS liver activity & \\
No reduction & $11 / 68(16.2 \%)$ \\
$25 \%$ & $12 / 68(17.6)$ \\
$\geq 50 \%$ & $45 / 68(66.2)$ \\
Reduction of MIS pancreatic activity & \\
No reduction & $3 / 55(5.5 \%)$ \\
$25 \%$ & $10 / 55(18.2 \%)$ \\
$\geq 50 \%$ & $42 / 55(76.4)$ \\
Reduction of robotic activity & \\
No reduction & $4 / 28(14.3)$ \\
$25 \%$ & $4 / 28(14.3)$ \\
$\geq 50 \%$ & $20 / 28(71.4 \%)$ \\
Hospital status & \\
Usual activity & $6 / 81(7.4 \%)$ \\
No elective benign surgery & $56 / 81(69.1 \%)$ \\
No any elective surgery & $15(18.5 \%)$ \\
Complete lockdown & $4(4.9 \%)$ \\
\hline &
\end{tabular}

\section{Outcome analysis}

Variables were processed and analyzed using IBM SPSS Statistics for Microsoft Windows version 22 (IBM Corp., Orchard Road Armonk, New York, US). Data were reported as number with percentage or as median with range (IQR). The recommendation was considered strong if $>80 \%$ of respondents agreed to it.

\section{Results}

\section{General details}

Eighty-one questionnaires were retrieved; the response rate was $93.5 \%$. In the 81 participating centers, 4.276 hepatic resections and 3.402 pancreatic resections were performed in 2019 , with a median of 63 (range 4-350) liver resections in 68 centers with hepatobiliary activity and 47 (range 5-450) pancreatic resections in 55 centers with pancreatic activity. 
Table 2 Minimally invasive HPB surgery during the COVID-19 pandemic

\begin{tabular}{|c|c|}
\hline \multicolumn{2}{|l|}{ Liver } \\
\hline MIS liver activity as a priority for referral to hub & $52 / 76(68.4 \%)$ \\
\hline MIS pancreas activity as a priority for referral to hub & $46 / 76(60.5 \%)$ \\
\hline \multicolumn{2}{|l|}{ Low complexity liver resection: switch MIS/open at hub center } \\
\hline Strongly agree or agree & $30 / 76(39.5 \%)$ \\
\hline Disagree or strongly disagree & $46 / 76(60.5 \%)$ \\
\hline \multicolumn{2}{|l|}{ Intermediate complexity liver resection: switch MIS/open at hub center } \\
\hline Strongly agree or agree & $40 / 75(53.3 \%)$ \\
\hline Disagree or strongly disagree & $35 / 75(46.7 \%)$ \\
\hline \multicolumn{2}{|l|}{ High complexity liver resection: switch MIS/open at hub center } \\
\hline Strongly agree or agree & $61 / 76(80.3 \%)$ \\
\hline Disagree or strongly disagree & $15 / 76(19.7 \%)$ \\
\hline \multicolumn{2}{|l|}{ Pancreas } \\
\hline \multicolumn{2}{|l|}{ Left pancreatectomy: switch MIS/open at hub center } \\
\hline Strongly agree & $37 / 76(48.7 \%)$ \\
\hline Disagree & $39 / 76(51.3 \%)$ \\
\hline \multicolumn{2}{|l|}{ Whipple procedure: switch MIS/open at hub center } \\
\hline Strongly agree & $64 / 75(85.3 \%)$ \\
\hline Disagree & $11 / 75(14.7 \%)$ \\
\hline \multicolumn{2}{|l|}{ General } \\
\hline \multicolumn{2}{|l|}{ Prioritization of patients based on: } \\
\hline Time from entry in the waiting list & $28 / 81(34.6 \%)$ \\
\hline Local resectability pattern & $39 / 81(48.1 \%)$ \\
\hline Biological aggressiveness & $60 / 81(74.1 \%)$ \\
\hline Alternative or bridging (including neoadjuvant chemo) treatments available or not & $50 / 81(61.7 \%)$ \\
\hline ASA score or Charlson Comorbidity Index & 23/81 (28.4) \\
\hline General performance status & $20 / 81(24.7 \%)$ \\
\hline \multicolumn{2}{|l|}{ Indication to MIS in SARS-CoV2 patients } \\
\hline Unmodified as per the timing and type of surgery required & $7 / 80(8.8 \%)$ \\
\hline Unmodified as per the timing and type of surgery required just if asymptomatic & $7 / 80(8.8 \%)$ \\
\hline Delayed in timing until SARS-CoV-2 negativization was proved & $66 / 80(82.5 \%)$ \\
\hline \multicolumn{2}{|l|}{ Technical variations in MIS } \\
\hline No & $19 / 74(25.7 \%)$ \\
\hline Yes: specific smoke aspirations & $36 / 74(48.6 \%)$ \\
\hline Yes: specific $\mathrm{CO} 2$ insufflations & $11 / 74(14.9 \%)$ \\
\hline Yes: specific attention to skin incisions appropriate to port dimensions & $23 / 74(31.1 \%)$ \\
\hline Yes: preference for trocars with balloon fixation & $25 / 74(33.8 \%)$ \\
\hline Yes: energy devices not used & $2 / 74(2.7 \%)$ \\
\hline Yes: pneumoperitoneal pressures kept at minimum & $28 / 74(37.8 \%)$ \\
\hline Yes: pneumoperitoneum aspiration before removal of trocars & $41 / 74(55.4 \%)$ \\
\hline Yes: minimization of time of Trendelenburg position & $6 / 74(8.1 \%)$ \\
\hline \multicolumn{2}{|l|}{ Fast-track protocol during COVID-19 } \\
\hline Yes, same indications & $58 / 78(74.4 \%)$ \\
\hline Yes, reduced number of indications & $15 / 78(19.2 \%)$ \\
\hline No, stopped & $5 / 78(6.4 \%)$ \\
\hline
\end{tabular}

The proportion of MIS procedures was $27.7 \%$ among hepatobiliary centers and $13 \%$ among pancreatic centers (data referred to year 2019). COVID-19 hub centres were $82.7 \%$ of the total.

\section{Waiting list management and indications}

As reported in Table 1, 72.8\% of centres are experiencing a reduction of routine elective HPB operations $\geq 50 \%$, being the institutional decision the most frequent reason 
for reduction (76.3\%). 71.4\% of centers performing robotic surgery reported a $\geq 50 \%$ reduction of activity. Among centres routinely performing MIS, $66.2 \%$ report a $\geq 50 \%$ reduction of minimally invasive liver resections. Similarly, $76.4 \%$ of pancreatic units report a $\geq 50 \%$ reduction of minimally invasive operations. For both hepatobiliary and pancreatic surgeries, this figure is motivated by institutional indication in most centres (70.9\%).

Centres for which the impact of COVID-19 pandemic on the waiting list management is considerable or severe are $40.7 \%$ and $29.6 \%$, respectively: in general, the waiting list managed mainly the setting priorities on specific criteria and/or referral to external hubs $(70 \%$ and $16.3 \%$, respectively). In $33.3 \%$ of centres, the process of prioritization is done on a case-by-case judgment, in $27.2 \%$, it is guided by institutional guidelines and in $23.5 \%$ by regional protocols. For oncologic patients, the most relevant factors to lead prioritization process are biological aggressiveness and the possibility of effective alternative or bridging treatments ( $74.1 \%$ and $61.7 \%$ of respondents). $39.5 \%$ of respondents agree with the switch from MIS to open based on the external hub evaluation in case of low complexity liver resection, and $48.7 \%$ for distal pancreatectomies. For intermediate and highly complex liver resections, the switch of approach is agreed from $53.4 \%$ to $80.2 \%$ of sending centres, respectively. Similarly, the proportion of strong agreement for the switch from MIS to open is $85.3 \%$ in Whipple procedures, as reported in Table 2. In the choice of the external hub for hepatobiliary and pancreatic surgery, MIS experience is considered as priority criterion by $68.4 \%$ and $60.5 \%$ of centres respectively. For SARS-CoV-2-positive patients elected to HPB operations, the opinion is for delaying in timing the indication to a MIS resection until viral negativization is proved $(82.5 \%$ of centres). Among the reasons, perceived risks for the OR staff and length of surgical procedure resulted the most relevant (53.3\% and $23.3 \%$ of respondents).

\section{Preoperative assessment}

$87.7 \%$ of surgeons suggest to test for virus positivity of all patients scheduled for elective surgery. Naso-faringeal swab is the investigation more frequently suggested (94.5\%), followed by thorax CT scan (34.2\%). For MIS resections, preoperative tests are judged almost unanimously as a mandatory prerequisite to proceed, with agreement by $88.7 \%$ of respondents.

\section{Intraoperative management}

As far as concerns, the protective measures during MIS surgery, $27.5 \%$ of centres apply COVID-19-specific measures for all the patients, whereas additional to the standard (but not COVID-19 specific) measures are taken in 47.5\% of centers. Within respondent centers, $32 \%$ indicate the presence of a dedicated operating room for SARS-CoV2-positive patients at their institution. $83.5 \%$ of respondents declare a preference for having an expert team (surgical/anesthesiological/nursing) during a MIS-HPB in patients with SARS-CoV-2 positivity. Various technological or technical variations with respect to the standard equipment are in general adopted by $74.3 \%$ of MIS centres, as detailed in Table 2 (pneumoperitoneum aspiration before removal of trocars: $55.4 \%$; specific smoke aspirations: $48.6 \%$; specific $\mathrm{CO}_{2}$ insufflations: $14.9 \%$; pneumoperitoneal pressure kept at minimum: $37.8 \%$; preference for trocars with balloon fixation: $33.8 \%$; specific attention to skin incisions appropriate to port dimension: $14.9 \%$; minimization of time of Trendelenburg position: $8.1 \%$; energy devices not used: $2.7 \%$ ). Regarding the acquisition of information on MIS during COVID-19 pandemic, Pubmed (or other libraries) is the main accredited source (82.5\% of respondents), followed by societies newsletters/ communications $(58.8 \%)$, and information reported from colleagues in Italy $(41.3 \%)$.

\section{Postoperative assistance of inpatients}

In the ward setting, to keep at minimum the number of involved persons given (72.5\%), to keep at the minimal physical distance (78.8\%), and to keep in-hospital working hours at essential for each member (78.8\%) are the most frequently adopted team protective measures.

The $74.4 \%$ of centres with an active fast-track protocol before the COVID-19 era have maintained its application for the same indications as before the pandemic, while the $19.2 \%$ apply the fast-track for a reduced number of indications. On average, the safety of a fast-track protocol during this epidemic is rated 9 (range 5-10). Before discharge, the majority of centres proceed with specific SARS-CoV-2 investigations only in case of positivity or clinical suspicion of infection $(86.4 \%)$.

\section{Human resources}

$19.8 \%$ of respondents suspect the infection of a member of the surgical staff during HPB surgery ( $8.8 \%$ during MIS). $21.3 \%$ of centres have at least one member of the surgical 
staff getting SARS-CoV-2 infection with a reasonable correlation to his/her contact with infected colleagues/patients during ward activities. Globally, $30 \%$ of centres are experiencing surgical staff shortage due to the SARS-CoV-2 infection of members.

\section{Discussion}

\section{Empty streets and empty (surgical) theatres during the pandemic. Get ready to work on priority}

The spread of COVID-19 in 2020 is leading to a significant slowing down of surgical activity, often dictated by hospital healthcare management $(72.8 \%$ of centers report $\geq 50 \%$ reduction of procedures, in $76.3 \%$ of cases secondary to a hospital management decision) with substantial negative impact on waiting lists ( $70.3 \%$ of centers have a considerable or severe impact on the waiting time because of the COVID-19 emergency). To achieve an adequate supply of care to patients with malignancies, $86.3 \%$ of centers have to reassess the prioritization criteria for any given case. Variables taken into account include time on waiting list, pattern of local resectability, biological aggressiveness of the disease, possibility for alternative or bridge treatments, age and ASA score, as well as general performance status (see Table 2 for details) for every case with the aim of stratifying the level of priority (prioritization process). In the highly affected area (Lombardy), the Regional Healthcare System government enforced the prioritization process, enabling centralization and monitoring the allocation of non-deferrable oncological cases based on the abovementioned specific criteria. In this area, all centers unable to offer timely surgery to oncological patients due to COVID-19-required reallocation of resources, received indications to refer high priority oncological patients to specific hub centers. Thirteen centers out of 81 participants $(16 \%)$ report referral of patients to hub centers: six referring surgeons took part in the surgical procedure at the hub centers by creating joint teams, while seven did not.

Despite attempts to develop strategies to avoid delays or even interruption of oncological care, the fragmentation of the National Healthcare System in regional and independent health systems seems to generate a large nationwide heterogeneity in prioritization management, which should be reported also in HPB surgery. Indeed, $16 \%$ of our respondents report absence of guidelines and $33.3 \%$ report the need for decision-making on a caseby-case basis. Regional or institutional guidelines are reportedly used only by $23.5 \%$ and $27.2 \%$ of respondents, respectively.

\section{Has the virus affected the commitment to minimally invasiveness?}

A nationwide effort for the implementation of minimally invasive programs for both hepatobiliary and pancreatic surgery was held in Italy in recent years, aiming to offer benefits over the open approach in selected cases while maintaining adequate standards of safety and oncological results [7, 8]. As a consequence, the median proportion of minimally invasive technique was $27.7 \%$ in liver and $13 \%$ in pancreas resections in 2019.

The COVID-19 pandemic seems to have hindered minimally invasive activity nationwide: $66.2 \%$ of centers report a reduction of minimally invasive liver resections $\geq 50 \%$; the same reduction is reported by $76.4 \%$ of pancreatic units. At the same time, access to robotic surgery was locked down in 16/24 units, where the approach is available. Interestingly, it is likely that the reduction in MIS activity actually parallels and is homogeneous with the overall reduction in oncological surgical activity. Indeed, despite reports in the available literature of increased risks for both patients and surgical staff when MIS is adopted in SARS-CoV-2-positive patients, the commitment of Italian HPB society towards MIS remains strong. Only $23.5 \%$ of respondents declare a change in the indications for minimally invasive approaches. These respondents adopt indications that are more restrictive and mainly applied to SARS-CoV-2-positive patients. In this group, the average proportion of patients switched from MIS to open hepatobiliary surgery is $30 \%$, and $50 \%$ for pancreatic resections.

It may be postulated that the commitment to minimal invasiveness could be maintained by the application of specific behaviors. Indeed, most answers to specific questions (would you suggest to test routinely all patients scheduled for elective surgery? How would you manage the indication to elective MIS HPB surgery in a patient with SARS-CoV-2 positivity?) agree to strongly recommended routine testing of all candidates to HPB surgery for SARS-CoV-2 (87.7\% agreement) and to delay MIS procedures upon SARS-CoV-2 negativization, if allowed by the clinical condition $(82.5 \%$ agreement).

In the choice of the external hub for hepatobiliary and pancreatic surgery, MIS experience is considered as priority criterion by $68.4 \%$ and $60.5 \%$ of centers, respectively. When the profile of technical complexity is low and, therefore, the MIS feasibility is high (low conversion rate), $60.5 \%$ of liver surgeons and $51.4 \%$ of pancreatic surgeons (for left pancreatectomy) disagree on a shift from MIS to open technique if proposed by the hub center. The rate of disagreement lowers 
inversely to an increasing profile of surgical challenge. In particular, for high complexity cases, $80.3 \%$ (liver) and $85.3 \%$ (Whipple) of surgeons agree with the possible switch from minimally invasive to open approach, giving thus priority to timely surgery without delay due to the willingness to address the case by MIS.

\section{Enhancing the safety of patients and surgeons...even without evidence}

Many concerns are raised regarding the possible increased risk of infection during MIS surgery in positive patients. Safety for both patients and surgical staff must be pursued as the primary endpoint of surgical treatment: consequently, all the available risk-limiting measures should be applied to contain viral dissemination $[9,10]$. Within respondent centers, $19.8 \%$ reported that staff member infections probably occurred during surgery, with $8.8 \%$ of infections being reported after MIS surgery.

Respondents to the present survey agree to recommend $(83.5 \%)$ the presence of a dedicated expert team in the operating theatre during MIS surgery for SARS-CoV-2-positive patients (or with unknown status). The procedure should ideally be performed in a room dedicated to COVID-19 patients. Respondents (87.1\%) recommend the use of technical variations in MIS surgery and in particular: pneumoperitoneum aspiration before removal of trocars; use of specific smoke aspirators; pneumoperitoneal pressures kept to a minimum; preference for trocars with balloon fixation; specific attention to skin incisions appropriate to port dimensions.

\section{Scientific background to face minimally invasive surgery during the pandemic: is it needed?}

Need for detailed information is a leitmotiv during an extraordinary event. It is, therefore, essential for healthcare providers to have full access to reliable sources to guide the decision-making process and to avoid unsafe practices. Indeed, all respondent express a discomfort in being culturally unprepared to face surgical activity during a situation of emergency when available resources have been downsized. Regarding the acquisition of information on MIS during COVID-19 pandemic, PubMed (or other libraries) was the main accredited source ( $82.5 \%$ of respondent), followed by societies newsletters/communications $(58,8 \%)$ and by the opinion of Italian colleagues $(41,3 \%)$. It is likely that the COVID-19 pandemic will come to its end soon. Anyway, the availability in the medical literature of recommendations and indications on healthcare system management within limited availability of resources will be a priceless tool in case of future crisis.

\section{Conclusions}

In Italian hospitals, minimally invasive surgery maintains its role despite the COVID-19 pandemic, with the registered reduction of cases being proportional to the overall reduction of the HPB surgical activity. The switch from minimally invasive to open technique can be considered as a valid option for cases with a high technical complexity. Several issues merit specific attention: screening for virus positivity should be universally performed; only expert surgical teams should operate on positive patients and specific technical measures to lower the biological risk of contamination during surgery must be followed. Future studies specifically designed to establish the true risks in minimally invasive surgery are suggested. Furthermore, a standard and univocal process of prioritization of patients from Regional Healthcare Systems is advisable.

Acknowledgements Collaborators (from AICEP): Mohammed Abu Hilal, Fondazione Poliambulanza, Brescia; Stefano Andrianello, Azienda Ospedaliera Universitaria, Verona; Alessandro Anselmo, Fondazione Policlinico Tor Vergata, Roma; Francesco Ardito, Fondazione Policlinico Universitario Gemelli, Roma; Luca Baiocchi Gian, Spedali Civili, Brescia; Filippo Banchini, Ospedale Guglielmo da Saliceto, Piacenza; Matteo Barabino, Ospedale San Paolo, Milano; Andrea Barberis, Ospedale Galliera, Genova; Claudio Bassi, Azienda Ospedaliera Universitaria, Verona; Giacomo Batignani, Azienda Ospedaliera Universitaria Careggi, Firenze; Carlo Battiston, Istituto Nazionale Tumori, Milano; Andrea Belli, Istituto Nazionale Tumori Fondazione Pascale, Napoli; Stefano Berti, ASL Spezzino, La Spezia; Paolo Bianco, Ospedale Pineta Grande, Castelvolturno; Alberto Brolese, Ospedale Santa Chiara, Trento; Stefania Brozzetti, Policlinico Umberto I, Roma; Giovanni Butturini, Ospedale P.Pederzoli, Peschiera del Garda; Fulvio Calise, Ospedale Pineta Grande, Castelvolturno; Kurt Carabott, Ospedale San Raffaele, Milano; Giovanni Capretti, Humanitas University \& Research Hospital, Rozzano; Riccardo Casadei, Policlinico Sant'Orsola Malpighi, Bologna; Matteo Cescon, Policlinico Sant'Orsola Malpighi, Bologna; Manuela Cesaretti, Azienda Ospedaliera Brotzu, Cagliari; Umberto Cillo, Azienda Ospedaliera Universitaria, Padova; Nicola Cinardi, ASP Catania, Catania; Michele Colledan, Ospedale Papa Giovanni XXIII, Bergamo; Alessandro Coppola, Università Campus Biomedico, Roma; Christian Cotsoglou, Ospedale di Vimercate, Vimercate; Stefano Crippa, Ospedale San Raffaele, Milano; Enrico Dalla Bona, Ospedali Riuniti, Ancona; Raffaele Dalla Valle, Azienda Ospedaliero Universitaria, Parma; Michela De Angelis, Azienda Ospedaliera Universitaria, Verona; Luciano De Carlis, Ospedale Niguarda, Milano; Fabrizio Di Benedetto, Policlinico di Modena, Modena; Pierluigi Di Sebastiano, Casa di Cura Pierangeli, Pescara; Laura Dova, ASL Spezzino, La Spezia; Giorgio Ercolani, Ospedale Pierantoni Morgagni, Forlì; Alessandro Esposito, Azienda Ospedaliera Universitaria, Verona; Maria Ettorre Giuseppe, Ospedale San Camillo Forlanini, Roma; Alberto Fabris, Azienda Ospedaliera Città della Salute e della Scienza, Torino; Alessandro Ferrero, Ospedale Mauriziano, Torino; Antonio Frena, Ospedale di Bolzano, Bolzano; Isabella Frigerio, Ospedale P.Pederzoli, Peschiera del Garda; Luca Gianotti, Ospedale San Gerardo, Monza; Antonio Giuliani, Università degli Studi del Molise, Campobasso; Felice Giuliante, Fondazione Policlinico Universitario Gemelli, Roma; Gianluca Grazi, Istituto Tumori Regina Elena, Roma; Enrico Gringeri, Azienda Ospedaliera Universitaria, Padova; Guido Griseri, Ospedale di Savona, Savona; 
Salvatore Gruttadauria, ISMETT, Palermo; Alfredo Guglielmi, Azienda Ospedaliera Universitaria, Verona; Francesco Izzo, Istituto Nazionale Tumori Fondazione Pascale, Napoli; Elio Jovine, Ospedale Maggiore, Bologna; Ezio Lanza, Humanitas University \& Research Hospital, Rozzano; Giuseppe Malleo, Azienda Ospedaliera Universitaria, Verona; Lorenzo Manzini, Azienda Unità Sanitaria Locale, Reggio Emilia; Marco Massani, Azienda Ospedaliera USL, Treviso; Vincenzo Mazzaferro, Istituto Nazionale Tumori, Milano; Riccardo Memeo, Policlinico Universitario, Bari; Francesco Minni, Policlinico Sant'Orsola Malpighi, Bologna; Luca Morelli, Ospedale Cisanello, Pisa; Gennaro Nappo, Humanitas University \& Research Hospital, Rozzano; Bruno Nardo, Ospedale dell'Annunziata, Cosenza; Francesco Orlando, Ospedale Cottolengo, Torino; Stefano Partelli, Ospedale San Raffaele, Milano; Alberto Patriti, Azienda Ospedaliera Ospedali Riuniti Marche Nord, Pesaro; Renato Patrone, Istituto Nazionale Tumori Fondazione Pascale, Napoli; Andrea Percivale, Ospedale Santa Corona, Pietra Ligure; Gaetano Piccolo, Ospedale di Lodi, Lodi; Matteo Ravaioli, Ospedale Sant'Orsola Malpighi, Bologna; Paolo Reggiani, Policlinico di Milano, Milano; Matteo Risaliti, Ospedale di Udine, Udine; Aldo Rocca, Università degli Studi del Molise, Campobasso; Renato Romagnoli, Ospedale Universitario Molinette, Torino; Fabrizio Romano, Ospedale San Gerardo, Monza; Nadia Russolillo, Ospedale Mauriziano, Torino; Andrea Ruzzenente, Azienda Ospedaliera Universitaria, Verona; Edoardo Saladino, Azienda Ospedaliera Papardo, Messina; Roberto Salvia, Azienda Ospedaliera Universitaria, Verona; Roberto Santambrogio, ASST Fatebenefratelli, Milano; Paola Tarchi, Ospedale Cattinara, Trieste; Albert Troci, ASST Fatebenefratelli, Milano; Roberto Troisi, Policlinico Federico II, Napoli; Lucio Urbani, Azienda Ospedaliero Universitaria Pisana, Pisa; Luigi Veneroni, Ospedale degli Infermi, Rimini; Giovanni Vennarecci, Ospedale Cardarelli, Napoli; Luca Vigano, Humanitas University \& Research Hospital, Rozzano; Massimo Viola, Ospedale Cardinale Giovanni Panico, Tricase; Fabio Vistoli, Ospedale Cisanello, Pisa; Marco Vivarelli, Ospedali Riuniti di Ancona, Ancona; Matteo Zanello, Ospedale Maggiore, Bologna; Giacomo Zanus, Ospedale di Treviso, Treviso; Alessandro Zerbi, Humanitas University \& Research Hospital, Rozzano.

\section{Compliance with ethical standards}

Conflict of interest The authors declare that they have no conflict of interest.

Research involving human participants and/or animals This article does not contain any studies involving animals performed by any of the authors. All procedures performed in human participants were in accordance with the ethical standards of the institutional research committee and with the 1964 Helsinki Declaration and its later amendments.

Informed consent Informed consent was obtained from all individual participants involved in the study.

\section{References}

1. do Nascimento IJB, Cacic N, Abdulazeem HM, von Groote TC, Jayarajah U, Weerasekara I, Esfahani MA, Civile VT, Marusic A, Jeroncic A, Carvas Junior N, Pericic TP, Zakarija-Grkovic I,
Guimarães SMM, Bragazzi NL, Bjorklund M, Sofi-Mahmudi A, Altujjar M, Tian M, Arcani DMC, O’Mathúna DP, Marcolino MS (2020) Novel Coronavirus Infection (COVID-19) in humans: a scoping review and meta-analysis. J Clin Med 9(4):E941. https ://doi.org/10.3390/jcm9040941

2. Zangrillo A, Beretta L, Silvani P, Colombo S, Scandroglio AM, Dell'Acqua A, Fominskiy E, Landoni G, Monti G, Azzolini ML, Monaco F, Oriani A, Belleti A, Sartorelli M, Pallanch O, Saleh O, Sartini C, Nardelli P, Lombardi G, Morselli F, Scquizzato T, Frontera A, Ruggeri A, Scotti R, Assanelli A, Dagna L, RovereQuerini P, Castagna A, Scarpellini P, Di Napoli D, Ambrosio A, Ciceri F, Tresoldi M (2020) Fast reshaping of intensive care unit facilities in a large metropolitan hospital in Milan, Italy: facing the COVID-19 pandemic emergency. Crit Care Resusc (Epub ahead of print)

3. Tuech JJ, Gangloff A, Di Fiore F, Michel P, Brigand C, Slim K, Pocard M, Schwarz L (2020) Strategy for the practice of digestive and oncological surgery during the Covid-19 epidemic. J Visc Surg. https://doi.org/10.1016/j.jviscsurg.2020.03.008

4. Ueda M, Martins R, Hendrie PC, McDonnell T, Crews JR, Wong TL, McCreery B, Jagels B, Crane A, Byrd DR, Pergam SA, Davidson NE, Liu C, Stewart FM (2020) Managing Cancer Care During the COVID-19 pandemic: agility and collaboration toward a common goal. J Natl Compr Canc Netw 20:1-4. https://doi. org/10.6004/jnccn.2020.7560

5. Spinelli A, Pellino G (2020) COVID-19 pandemic: perspectives on an unfolding crisis. Br J Surg. https://doi.org/10.1002/ bjs. 11627

6. Zheng MH, Boni L, Fingerhut A (2020) Minimally invasive surgery and the novel coronavirus. Outbreak lessons learned in China and Italy. Ann Surg. https://doi.org/10.1097/SLA.0000000000 003924

7. Aldrighetti L, Ratti F, Cillo U, Ferrero A, Ettorre GM, Guglielmi A, Giuliante F, Calise F, Italian Group of Minimally Invasive Liver Surgery (I GO MILS) (2017) Diffusion, outcomes and implementation of minimally invasive liver surgery: a snapshot from the I Go MILS (Italian Group of Minimally Invasive Liver Surgery) Registry. Updates Surg 69(3):271-283

8. Capretti G, Boggi U, Salvia R, Belli G, Coppola R, Falconi M, Valeri A, Zerbi A (2019) Application of minimally invasive pancreatic surgery: an Italian survey. Updates Surg 71(1):97-103

9. Aminian A, Safari S, Razeghian-Jahromi A, Ghorbani M, Delaney CP (2020) COVID-19 outbreak and surgical practice: unexpected fatality in perioperative period. Ann Surg. https://doi.org/10.1097/ SLA.0000000000003925

10. Ngoi N, Lim J, Ow S, Jen WY, Lee M, Teo W, Ho J, Sundar R, Tung ML, Lee YM, Ngo E, Lim SW, Ong J, Lim F, Bonney G, Vellayappan B, Ho F, Tey J, Chan N, de Mel S, Poon M, Lee SY, Koh LP, Liu TC, Tan LK, Wong A, Wong A, Lim SE, Yeoh A, Wong JE, Tan D, Goh BC, Chng WJ, Soo R, Chee CE, Chee YL, Lee SC, Jeyasekharan AD, National University Cancer Institute, Singapore (NCIS) (2020) A segregated-team model to maintain cancer care during the COVID-19 outbreak at an Academic Center in Singapore. Ann Oncol. https://doi.org/10.1016/j.annon c.2020.03.306

Publisher's Note Springer Nature remains neutral with regard to jurisdictional claims in published maps and institutional affiliations. 